\title{
Introducing TOPMAST, the first double-blind randomized clinical trial specifically dedicated to perioperative maintenance fluid therapy in adults
}

\author{
Steven Hendrickx ${ }^{1,2}$, Karen Van Vlimmeren ${ }^{1,2}$, Ingrid Baar ${ }^{1}$, Walter Verbrugghe ${ }^{1}$, Karolien Dams ${ }^{1}$, \\ Sophie Van Cromphaut ${ }^{1}$, Ella Roelant ${ }^{3}$, Bart Embrecht ${ }^{2}$, Anouk Wittock ${ }^{2}$, Pieter Mertens ${ }^{2}$, \\ Jeroen M. Hendriks ${ }^{4}$, Patrick Lauwers ${ }^{4}$, Paul E. Van Schil ${ }^{4}$, Amaryllis H. Van Craenenbroeck ${ }^{5}$, \\ Tim Van den Wyngaert ${ }^{6,7}$, Philippe G. Jorens ${ }^{1,7}$, Niels Van Regenmortel ${ }^{1,8}$ \\ ${ }^{1}$ Department of Intensive Care Medicine, Antwerp University Hospital, Edegem (Antwerp), Belgium \\ ${ }^{2}$ Department of Anesthesiology, Antwerp University Hospital, Antwerp, Belgium \\ ${ }^{3}$ Clinical Trial Center (CTC), Clinical Research Center Antwerp, Antwerp University Hospital, University \\ of Antwerp, Antwerp, Belgium \\ ${ }^{4}$ Department of Thoracic and Vascular Surgery, Antwerp University Hospital, Antwerp, Belgium \\ ${ }^{5}$ Department of Nephrology, Antwerp University Hospital, Antwerp, Belgium \\ ${ }^{6}$ Department of Nuclear Medicine, Antwerp University Hospital, Antwerp, Belgium \\ ${ }^{7}$ Faculty of Medicine and Health Sciences, University of Antwerp, Antwerp, Belgium \\ ${ }^{8}$ Department of Intensive Care Medicine, Ziekenhuis Netwerk Antwerpen, Campus Stuivenberg, Antwerp, Belgium
}

\begin{abstract}
Background: Although prescribed to every patient undergoing surgery, maintenance fluid therapy is a poorly researched part of perioperative fluid therapy. The tonicity of the chosen solutions, could be an important cause of morbidity, with hyponatremia being a potential side effect of hypotonic solutions, where isotonic solution could lead to fluid overload. Methods: The TOPMAST-trial is an ongoing prospective single-center double-blind randomized trial comparing an isotonic and a hypotonic maintenance fluid strategy during and after surgery in patients undergoing different types of major thoracic surgery. Patients receive $\mathrm{NaCl} 0.9 \%$ in glucose $5 \%$ with an added $40 \mathrm{mmol} \mathrm{L}^{-1}$ of potassium chloride or a premixed solution containing $54 \mathrm{mmol} \mathrm{L}^{-1}$ sodium, $55 \mathrm{mmol} \mathrm{L}^{-1}$ chloride and $26 \mathrm{mmol}$ of potassium at a rate of $27 \mathrm{~mL}$ per $\mathrm{kg}$ of body weight per day. The primary hypothesis is that isotonic maintenance solutions cause a more positive perioperative fluid balance than hypotonic fluids. Different secondary safety endpoints will be explored, especially the effect of the study treatments on the occurrence electrolyte disturbances (e.g. hyponatremia, hyperchloremia) and a set of clinical endpoints. Efficacy endpoints include the need for resuscitation fluids and assessment of renal and hormonal adaptive mechanisms. An anticipated 68 patients will be included between March 2017 and January 2018. Discussion: The study will provide the most comprehensive evaluation of clinically important outcomes associated with the choice of perioperative maintenance fluid therapy.
\end{abstract}

Anaesthesiology Intensive Therapy 2017, vol. 49, no 5, 366-372

Trial registration: ClinicalTrials.gov NCT03080831

Key words: maintenance fluid therapy, fluid balance, fluid overload, electrolyte disorders, hyponatremia, hyperchloremia

Until the early 2000s, perioperative fluid management was predominantly based upon the experience, training and even personal convictions of the prescribing anesthesiologists and surgeons. During the past few years, an increased interest in perioperative goal directed therapy lead to a more restric- 
tive use of resuscitation fluids during surgery [1]. The potentially detrimental effects of chloriderich resuscitation fluids such as $\mathrm{NaCl} 0.9 \%$ were brought under the clinician's attention and lead to an increased interest in chloride-poor balanced solutions [2-4]. One important, but substantial part of perioperative fluid therapy remained largely unresearched, though: maintenance fluid therapy, prescribed to cover the patient's need for fluid and electrolytes during the period he or she is unable to ingest food or fluid. The only research on this part of fluid therapy was performed in the field of pediatric medicine and showed a higher incidence of hyponatremia using hypotonic solutions [5]. The subsequent recommendation to avoid hypotonic maintenance solution remains heavily debated [6-8]. It is also unknown whether these findings could be extrapolated to an adult population. On the other hand, a recent study performed by our group in healthy volunteers demonstrated an unintentional plasma expansion and subsequent fluid retention of around $600 \mathrm{~mL}$ under maintenance fluid therapy using $\mathrm{NaCl} 0.9 \%$ in glucose $5 \%$ with $40 \mathrm{mmol} \mathrm{L}^{-1}$ of potassium chloride versus a premixed hypotonic solutions containing only $54 \mathrm{mmol} \mathrm{L}^{-1}$ of sodium [9]. Also, hyperchloremia was frequently encountered in the isotonic treatment arm, while no hyponatremia could be demonstrated under hypotonic treatment. It is unclear whether these findings could be reproduced in a clinical setting, which could be especially important in surgical patients where it was clearly demonstrated that fluid-induced weight gain leads to perioperative morbidity [10].

The primary hypothesis of this study is that isotonic compared to hypotonic maintenance fluid therapy at a guidelinerecommended rate leads to a more positive perioperative fluid balance after major surgery in adults. Furthermore, we wanted to explore the effect of the tonicity and the composition of maintenance fluid therapy on the serum concentrations of sodium, chloride, potassium, calcium, magnesium and phosphate and on the incidence of disorders of these electrolytes, particularly hyponatremia, hyperchloremia. Different clinical secondary endpoints will be explored as additional safety endpoints. We also aimed to explore different physiological adaptive mechanisms and markers of volume status (aldosterone and fractional excretion of sodium).

\section{METHODS}

Tonicity of Perioperative Maintenance Solutions in Thoracic Surgery (TOPMAST) is a prospective single-center double-blind randomized trial comparing the clinical outcomes of adult patients undergoing major surgery with two different maintenance solutions.

\section{INCLUSION AND EXCLUSION CRITERIA}

The study is being conducted at the Antwerp University Hospital, a tertiary care academic hospital in Belgium. Eligible patients (at least 18 years of age) are scheduled for elective thoracic surgery for presumed or proven lung cancer: pulmonary lobectomy, bilobectomy or segmentectomy, through thoracotomy or video-assisted or robot-assisted thoracoscopy. Patients undergoing pneumonectomy are

Table 1. Summary of exclusion criteria

\begin{tabular}{|c|c|}
\hline Reason for exclusion & Exclusion criterion \\
\hline \multirow{9}{*}{$\begin{array}{l}\text { Difficulty in interpretation } \\
\text { of the primary and } \\
\text { secondary endpoints }\end{array}$} & Estimated glomerular filtration rate (eGFR) lower than $60 \mathrm{~mL} \mathrm{~min} \mathrm{~min}^{-1} 1.73 \mathrm{~m}^{-2}$ (CKD-EPI) [11] \\
\hline & Body mass index (BMI) lower than $15 \mathrm{~kg} \mathrm{~m}^{-2}$ or exceeding $40 \mathrm{~kg} \mathrm{~m}^{-2}$ \\
\hline & Chronic diuretic or desmopressin use \\
\hline & Acute or chronic liver failure \\
\hline & Pregnancy \\
\hline & Treatment with enteral or parenteral feeding \\
\hline & Initiation of massive transfusion protocol during surgery (post-hoc) \\
\hline & Need for resuscitation with $>2.5 \mathrm{~L}$ of fluids during surgery (post-hoc exclusion) \\
\hline & Development of acute kidney injury (post-hoc exclusion from analysis of primary endpoint only) \\
\hline \multirow{5}{*}{$\begin{array}{l}\text { Potential harm due to } \\
\text { study solutions }\end{array}$} & History of heart failure NYHA III-IV \\
\hline & Brittle insulin-dependent diabetes mellitus \\
\hline & $\begin{array}{l}\text { Neurological contra-indication to hypotonic fluids (cerebrovascular accident, traumatic brain injury or intracranial } \\
\text { hemorrhage within two weeks before surgery) }\end{array}$ \\
\hline & The syndrome of inappropriate secretion of antidiuretic hormone (SIADH) \\
\hline & $\begin{array}{l}\text { Electrolyte disorders during preoperative checkup: sodium level below } 130 \mathrm{mmol} \mathrm{L}^{-1} \text { or exceeding } 150 \mathrm{mmol} \mathrm{L}^{-1} \text { or } \\
\text { a potassium level above } 5 \mathrm{mmol} \mathrm{L}^{-1}\end{array}$ \\
\hline
\end{tabular}


Table 2. Detailed study fluid composition

\begin{tabular}{lc}
\hline $\begin{array}{l}\text { Isotonic Fluid: } \mathrm{NaCl} 0,9 \% \text { in glucose } 5 \% \text { supplemented } \\
\text { by } 40 \mathrm{mmol} \mathrm{L}^{-1} \mathrm{KCl}\end{array}$ \\
\hline Sodium & $154 \mathrm{mmol} \mathrm{L}^{-1}$ \\
Chloride & $194 \mathrm{mmol} \mathrm{L}^{-1}$ \\
Potassium & $40 \mathrm{mmol} \mathrm{L}^{-1}$ \\
Osmolarity & $614 \mathrm{mOsm} \mathrm{L}^{-1}$ \\
Tonicity & $373 \mathrm{mmol} \mathrm{L}^{-1 *}$ \\
Strong ion difference [13] & $0 \mathrm{mEq} \mathrm{L}^{-1}$ \\
Hypotonic Fluid: Glucion $5 \%{ }^{\circ}$ supplemented by & $40 \mathrm{~mL} \mathrm{water} \mathrm{for}^{-1}$ \\
blinding reasons & $54 \mathrm{mmol} \mathrm{L}^{-1}$ \\
Sodium & $55 \mathrm{mmol} \mathrm{L}^{-1}$ \\
Chloride & $26 \mathrm{mmol} \mathrm{L}^{-1}$ \\
Potassium & $6.2 \mathrm{mmol} \mathrm{L}^{-1}$ \\
Phospate & $2.6 \mathrm{mmol} \mathrm{L}^{-1}$ \\
Magnesium & $25 \mathrm{mmol} \mathrm{L}^{-1}$ \\
Lactate & $430 \mathrm{mOsm} \mathrm{L}^{-1}$ \\
Osmolarity & $162 \mathrm{mmol} \mathrm{L}^{-1 * *}$ \\
Tonicity & $\pm 30 \mathrm{mEq} \mathrm{L}^{-1}$ \\
\hline Strong ion difference & \\
\hline
\end{tabular}

* $154+194+40 \mathrm{mmol} / 1.040 \mathrm{~L}$

** $52+55+26+6,2+2,6+25 \mathrm{mmol} / 1.040 \mathrm{~L}$

excluded because of the need for a restrictive fluid strategy during and after this type of surgery. Other exclusion criteria are summarized in Table 1 and include conditions that could potentially complicate the interpretation of the results or pose a risk to the patient.

\section{STUDY FLUIDS}

Patients are randomly allocated to receive one of the two different study fluids (Table 2). The first fluid is glucose $5 \%$ in $\mathrm{NaCl} 0.9 \%$ with an added $40 \mathrm{mmol}$ of potassium chloride, so that the prescribed fluid volume would represent a guideline-recommended daily dose of potassium of around $1 \mathrm{mmol}$ per kg of body weight per day) [12]. Notably, the addition of potassium chloride essentially renders this solution hypertonic, although potassium has a negligible effect on tonicity after administration. The second fluid is a commercially available premixed solution (Glucion $5 \%{ }^{\circ}$, Baxter Healthcare, Deerfield Illinois, USA) containing amongst others $54 \mathrm{mmol} \mathrm{L}^{-1}$ of sodium, $26 \mathrm{mmol} \mathrm{L}^{-1}$ of potassium and $55 \mathrm{mmol} \mathrm{L}^{-1}$ of chloride. The study fluids are administered at a guideline-recommended rate of $27 \mathrm{~mL}$ per kg of body weight per day with a maximum of $100 \mathrm{~mL} \mathrm{~kg}^{-1}$ day $^{-1}$, according to current guidelines $\left(25-30 \mathrm{~mL} \mathrm{~kg}^{-1} \mathrm{day}^{-1}\right)$.

Treatment allocation is concealed from the study investigators, treating physicians, nurses and patients, and is performed by an independent collaborator who is not involved in the care of the patient or the analysis of the results. Because subjects would need different amounts of fluid volume depending on their BMI, the preparation of persubject study fluids was deemed too complex. Alternatively, solutions are therefore batch-prepared in two random pairs (A to D) using opaque bags that are identical in appearance. Urgent unblinding of the study treatment remains possible in case of an emergency. For reasons of preservation it is impossible to add potassium chloride to the first study fluid at the time of blinding. Since potassium chloride needs to be added to only one of the study fluids, we concomitantly blinded single-use containers with water for injection, visually identical to the ones containing potassium chloride, to be added to the hypotonic study fluid by the treating physician or nursing staff. During the study, the individual fluid bags are marked with the subject's data and collected for a double check immediately after completing the study treatment by an independent collaborator.

\section{INFORMED CONSENT, RANDOMIZATION PROCESS AND INTERVENTION}

The day before surgery, potential subjects are informed of the study by a member of the study team, are handed an information brochure and provide written informed consent. Subjects are randomized (1:1), stratified based on their body weight (lower or higher than $74 \mathrm{~kg}$, based on the median of a historical cohort), as body weight is an important determinant of the amount of study fluid to be received. The randomization schedule is electronically generated by permuted block randomization with a block size of 2 . Figure 1 shows the detailed study outline, including the sampling schedule of serum, fresh urine (to calculate fractional excretion of sodium) and collected urine (to calculate measured GFR [mGFR] and sodium balance). All serum samples are also stored for possible additional assessments (e.g. neutrophil gelatinase-associated lipocalin [NGAL]).

Subjects routinely refrain from solid food intake from the night before surgery. They are invited to drink up to two hours before being called to the operating theater. Upon arrival, patient controlled epidural analgesia (PCEA) is applied using a thoracic epidural catheter. Any fluids necessary to treat possible hypotension are precisely registered. After the induction of general aenesthesia, a fresh urine sample for sodium and creatinine is collected, serum is sampled for sodium, potassium, chloride, calcium, phosphate, magnesium, creatinine, albumin and aldosterone. At $\left(\mathrm{T}_{0}\right)$ a bladder catheter is placed, the bladder is voided, and the study fluid is started after being supplemented with the blinded additive (potassium chloride or water for injection). All urine produced during surgery is collected. Resuscitation fluids, blood products and additional electrolytes are administered at the discretion of the treating anaesthesiologist and are recorded. The time of initiation of PCEA ( $\left.T_{\text {pcea }}\right)$, intubation ( $\left.T_{\text {int }}\right)$, 


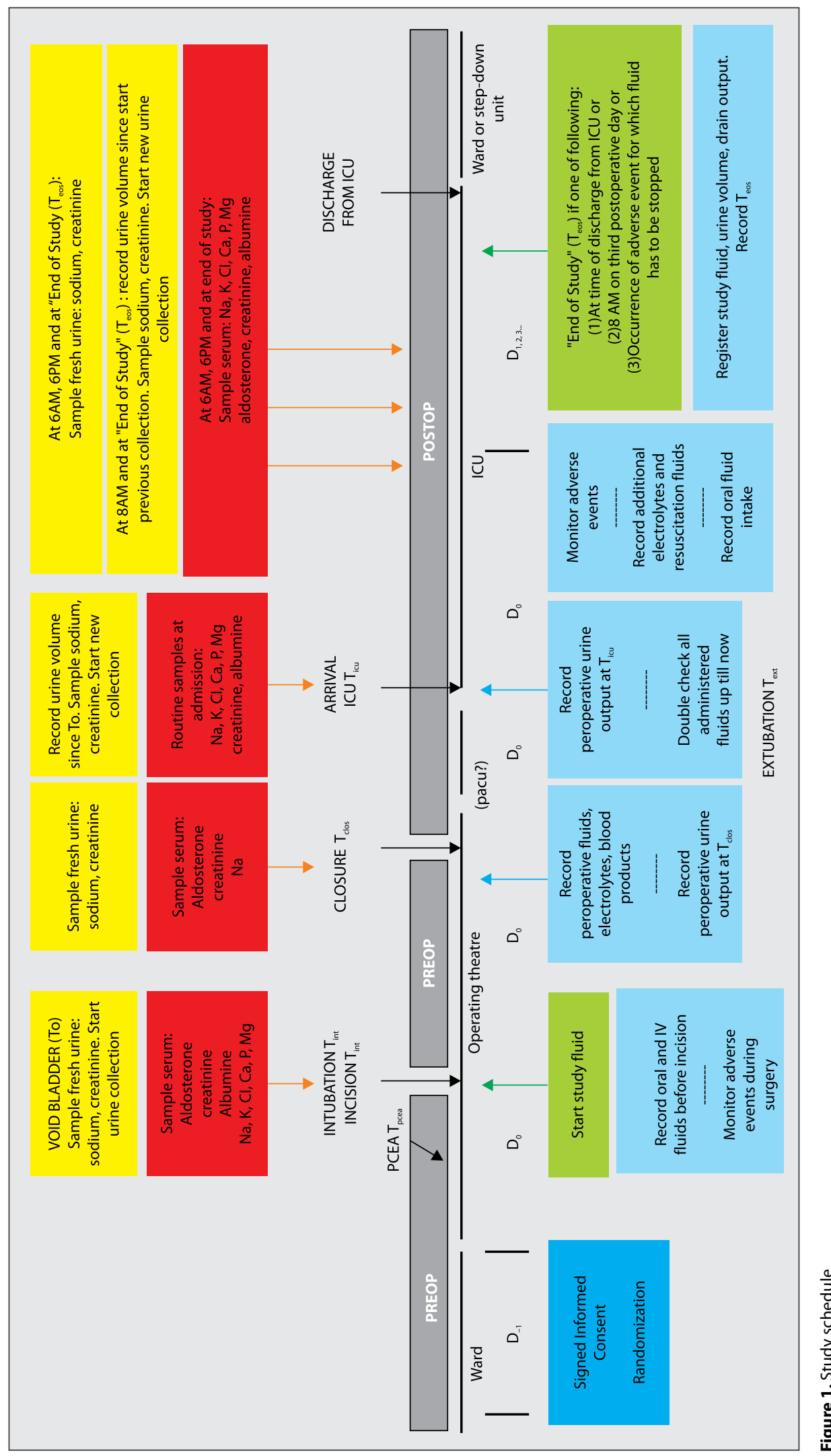


incision $\left(T_{\text {inc }}\right)$, closure $\left(T_{\text {clos }}\right)$ and extubation $\left(T_{\text {ext }}\right)$ are also recorded. At the end of surgery, fresh urine is sampled for sodium and creatinine and serum for aldosterone, sodium and creatinine. If possible, the patient is weaned from the ventilator before leaving the operating theatre. If a short stay on in the post anaesthesia care unit (PACU) is necessary, study treatment is continued there and required additional resuscitation fluids are recorded. Subsequently, the patient is transferred to the intensive care unit. Upon arrival in the ICU, the additional fluids (routinely 1,000-1,500 mL of PlasmaLyte ${ }^{\bullet}$, Baxter Healthcare, Deerfield Illinois, USA) used during surgery are reviewed by the anaesthesiologist and the intensivist. Every infusion treatment that is started during surgery needs to be administered fully to allow for precise recording of administered volume. The volume of urine that is produced since $\mathrm{T}_{0}$ is recorded and sampled for sodium and creatinine. At the same time, serum samples are taken (sodium, potassium, chloride, calcium, phosphate, magnesium, creatinine, albumin) as routinely performed at admission to the ICU.

In the ICU, the study treatment is administered at an unchanged rate until the end of the study ("End of Study" $\left.\left[T_{\text {eos }}\right]\right) . T_{\text {eos }}$ is reached from the moment one of the following events occurs: 1) the patient is discharged from the ICU; 2) an adverse event occurs for which the treating physician decides the study fluid needs to be stopped (see below), or 3) $8 \mathrm{AM}$ on the third postoperative day is reached while the patient is still admitted to the ICU. In the meantime, every day of ICU stay at 6 AM and 6 PM fresh urine (sodium, creatinine) and serum samples (sodium, potassium, chloride, calcium, phosphate, magnesium, creatinine, albumin and aldosterone) are obtained. At $8 \mathrm{AM}$ the amount of urine produced since the last urine collection is recorded and sampled for sodium and creatinine. At $\mathrm{T}_{\text {eos' }}$, the volume of the final urine collection is recorded and sampled for sodium and creatinine, and final samples of fresh urine (sodium, creatinine) and serum are obtained (sodium, potassium, chloride, calcium, phosphate, magnesium, creatinine, albumin and aldosterone). During the ICU stay, the treating physician can prescribe resuscitation fluids, correct hypokalemia or transfuse blood products, but the amount and the reason for administration have to be recorded. The patient is also allowed to drink; these fluids are carefully registered. Potential adverse events are monitored closely and can lead to the discontinuation of the study fluid.

\section{ADVERSE REACTIONS AND ADVERSE EVENTS}

Both study fluids are used in maintenance fluid therapy, daily and globally, in and outside the perioperative setting. Therefore, the odds of experiencing adverse reactions attributable to the study solutions are extremely small. Some preexisting conditions could however be aggravated by the tonicity, potassium and glucose content of the study fluids, especially at the rate at which they are administered. Therefore, we excluded certain conditions, such as heart, kidney and liver failure, electrolyte disorders, neurological conditions leading to brain edema and brittle diabetes. Rare, but known side effects such as urticaria or more serious anaphylactic reaction, local irritation, pain, phlebitis or thrombophlebitis are to be treated accordingly by the treating clinicians. Also, the decision to stop the study treatment lies with the treating physician, after which urine volume is recorded and the necessary closing samples at $\mathrm{T}_{\text {eos }}$ are taken.

A few (serious) adverse events can be related to the study treatment and trigger the end of a subject's study period $\left(T_{\text {eos }}\right)$. Evolving hyponatremia below $130 \mathrm{mmol} \mathrm{L}^{-1}$, hyperkalemia $>5 \mathrm{mmol} \mathrm{L}^{-1}$ (after pseudo-hyperkalemia has been ruled out), important hyperglycemia (> $350 \mathrm{mg}$ $\mathrm{dL}^{-1}$ ) are considered valid triggers of the end of the study period. Also, the clinical or radiographical occurrence of pulmonary edema or an otherwise unexplained increase in the need for oxygen, can be reasons for the treating clinicians to stop the study fluid. The use of diuretics renders the interpretation of primary and secondary endpoints impossible and immediately triggers $\mathrm{T}_{\text {eos }}$. Clinicians are therefore encouraged to use diuretic therapy only in the case of clinically relevant fluid overload and not for oliguria. As oliguria is one of the points of interest of the study and could be - per hypothesis - be related to the study treatment, treating clinicians are encouraged to prescribe resuscitation fluids for oliguria only when urine production decreased below $0.5 \mathrm{~mL}$ per kg of body mass per hour for at least 6 consecutive hours. All other serious adverse events (cardiopulmonary resuscitation, major bleeding, respiratory failure without congestion, the need for urgent surgical revision) are considered unrelated to the study, although the discontinuation of the study treatment remains at the discretion of the treating physician.

\section{SAMPLE SIZE AND ANALYSIS}

The power analysis is based on the results of the $\mathrm{MIH}$ MoSA experiment [9]. We determined that a sample size of 68 patients would provide a power of $80 \%$ to detect a difference in fluid balance of $595 \mathrm{cc}$ after $48 \mathrm{~h}$ at an alpha set at 0.05 with an assumption of a $10 \%$ dropout rate. Due to the potential disproportional impact on fluid balance of massive transfusion (defined as the initiation of the hospital's massive transfusion protocol), unexpected cardiopulmonary bypass during surgery or the development of acute kidney injury before $T_{\text {eos' }}$ subjects that experience one of these events are considered dropouts and are excluded from the per-protocol analysis. Analyses will also be conducted on an intention-to-treat basis. Continuous outcomes will be compared with the use of mean differences and unpaired 
t-tests and binary outcomes using relative risks using with 95\% confidence intervals and chi-square tests.

We collect the following data on patient characteristics: age, gender, body weight, and length, body mass index, SAPS-3 score at ICU admission and the estimated glomerular filtration rate [11]. Regarding the surgical procedure, we collect data on the type of surgery, the surgical technique (thoracostomy, video-assisted or robot-assisted thoracoscopy), blood loss during surgery, the amount of vasopressors used during surgery and the duration of the surgery and mechanical ventilation (as positive pressure ventilation could induce fluid retention).

The primary endpoint of the study is net fluid balance, calculated as the sum of all intravenous and oral intake minus urine production, and expressed in $\mathrm{mL}$ per $\mathrm{kg}$ of body weight, adjusted for the duration of the study period. As secondary endpoints we distinguish safety endpoints and efficacy endpoints. The following secondary safety endpoints were selected: the occurrence of hyponatremia (defined as a serum sodium measurement [excluding point-of-care measurements in view of the low limits of agreement] below the minimum of the normal range [135 $\left.\mathrm{mmol} \mathrm{L}^{-1}\right]$ with a decrease of at least $3 \mathrm{mmol} \mathrm{L}^{-1}$ from the baseline measurement, to account for already hyponatremic patients and laboratory measurement error); hypernatremia (comparably defined as a serum sodium measurement above the maximum of the normal range [145 $\left.\mathrm{mmol} \mathrm{L}^{-1}\right]$ with an increase of at least $3 \mathrm{mmol} \mathrm{L}^{-1}$ from the baseline measurement); hypokalemia $\left(<3.5 \mathrm{mmol} \mathrm{L}^{-1}\right.$ and a decrease of at least $0.2 \mathrm{mmol} \mathrm{L}^{-1}$ or the administration of additional potassium during the study); hyperkalemia in the absence of hemolysis ( $>5.1 \mathrm{mmol} \mathrm{L}^{-1}$ and an increase of at least $0.2 \mathrm{mmol} \mathrm{L}^{-1}$ ); hyperchloremia (> $109 \mathrm{mmol} \mathrm{L}^{-1}$ and an increase of at least $\left.2 \mathrm{mmol} \mathrm{L}^{-1}\right)$; hypochloremia $\left(<101 \mathrm{mmol} \mathrm{L}^{-1}\right.$ and a decrease of at least $\left.2 \mathrm{mmol} \mathrm{L}^{-1}\right)$; hypophosphatemia $\left(<0.81 \mathrm{mmol} \mathrm{L}^{-1}\right.$ and a decrease of at least $0.2 \mathrm{mmol} \mathrm{L}^{-1}$ or the administration of additional phosphate); hyperphosphatemia ( $>1.45 \mathrm{mmol} \mathrm{L}^{-1}$ and an increase of at least $\left.0.2 \mathrm{mmol} \mathrm{L}^{-1}\right)$; hypocalcemia $(<2.15$ $\mathrm{mmol} \mathrm{L}^{-1}$ and a decrease of at least $0.2 \mathrm{mmol} \mathrm{L}^{-1}$ or the administration of additional calcium) and hypercalcemia ( $>2.55 \mathrm{mmol} \mathrm{L}^{-1}$ and an increase of at least $0.2 \mathrm{mmol} \mathrm{L}^{-1}$ ). We further consider the mean difference from the baseline value of the abovementioned electrolytes. On a clinical level, we consider the highest $\mathrm{paO}_{2} / \mathrm{FiO}_{2}$ ratio during the study period, the occurrence of new onset atrial fibrillation, the occurrence of pulmonary edema for which the study fluid had to be stopped and the mGFR on urine collections with a duration of at least 12 hours. We selected the following efficacy endpoints: 1) the effect of the study fluid on amount of additional resuscitation fluids; 2 ) the effect of the study fluid on volume status by assessing adaptational mechanisms: change from baseline aldosterone level, urinary sodium and fractional excretion of sodium; 3 ) the effect of study fluid on sodium balance (calculated as the cumulative amount of sodium by the use of all intravenous fluids (study fluids and non-study fluids) minus cumulative amount of urinary sodium excretion.

\section{CONCLUSION}

In summary, the TOPMAST-trial is an ongoing prospective single-center double-blind randomized trial comparing an isotonic and a hypotonic maintenance fluid strategy. It is the first trial to look into this important part of fluid therapy. The first patient was enrolled on March $6^{\text {th }}, 2017$, and enrollment is scheduled for completion by the end of 2017. Upon completion, the TOPMAST-trial will provide comprehensive data on the comparative clinical effects of an isotonic versus a hypotonic maintenance fluid strategy in the perioperative setting. Given the extremely broad use of maintenance fluids and the ongoing debate on the potentials hazards of both hypotonic solutions (hyponatremia) and isotonic solutions (fluid overload), the results are important to the many clinicians treating patients undergoing major surgery.

\section{ACKNOWLEDGEMENTS}

1. Source of funding - the study is funded by an unrestricted educational grant by Baxter Healthcare, Deerfield Illinois, USA. The funding source has no role in the design and conduct of the trial and is not involved in the analysis, interpretation and report of the data.

2. We are indebted to the medical, paramedical and administrative staff of the department of anesthesiology, thoracic and vascular surgery and intensive care of the Antwerp University Hospital, whose continued teamwork was essential to the preparation and conduct of this study.

3. Conflicts of interest: NVR has received speaker's fees from Baxter Belgium and resided in a medical advisory board organized by Baxter Healthcare, US. He is also the co-founder and member of the executive board of the International Fluid Academy, a not-for-profit organization promoting education on fluid management and hemodynamic monitoring that received sponsoring from the industry (www.fluidacademy.org). The other authors have no possible conflicts of interest in relation to this article.

4. Trial registration. The study is approved by the Institutional Review Board of the Antwerp University Hospital (Directive 17/4/34) and the relevant national authority - the Federal Agency for Medicines and Health Products, Belgium (EudraCT 2017-000450-20). It is an investigator-initiated trial that was registered with ClinicalTrials.gov (NCT03080831) prior to the start of patient enrollment. 


\section{References:}

1. Bennett VA, Cecconi M. Perioperative fluid management: From physiology to improving clinical outcomes. Indian J Anaesth. 2017; 61 (8): 614-621, doi: 10.4103/ija.IJA_456_17, indexed in Pubmed: 28890555.

2. Yunos NM, Bellomo R, Hegarty C, et al. Association between a chloride-liberal vs chloride-restrictive intravenous fluid administration strategy and kidney injury in critically ill adults. JAMA. 2012; 308(15): 1566-1572, doi: 10.1001/jama.2012.13356, indexed in Pubmed: 23073953.

3. Chowdhury AH, Cox EF, Francis ST, et al. A randomized, controlled, double-blind crossover study on the effects of 2 -L infusions of $0.9 \%$ saline and plasma-lyte ${ }^{\oplus} 148$ on renal blood flow velocity and renal cortical tissue perfusion in healthy volunteers. Ann Surg. 2012; 256(1): 18-24, doi: 10.1097/SLA.0b013e318256be72, indexed in Pubmed: 22580944.

4. Young P, Bailey M, Beasley R, et al. SPLIT Investigators, ANZICS CTG. Effect of a buffered crystalloid solution vs saline on acute kidney injury among patients in the intensive care unit:The SPLIT randomized clinical trial. JAMA. 2015; 314(16): 1701-1710, doi: 10.1001/jama.2015.12334, indexed in Pubmed: 26444692.

5. McNab S, Duke T, South $\mathrm{M}$, et al. $140 \mathrm{mmol} / \mathrm{L}$ of sodium versus $77 \mathrm{mmo}$ $\mathrm{I} / \mathrm{L}$ of sodium in maintenance intravenous fluid therapy for children in hospital (PIMS): a randomised controlled double-blind trial. Lancet. 2015; 385(9974): 1190-1197, doi: 10.1016/S0140-6736(14)61459-8, indexed in Pubmed: 25472864.

6. Moritz ML, Ayus JC. Maintenance intravenous fluids in acutely ill patients. N Engl J Med. 2015; 373(14): 1350-1360, doi: 10.1056/NEJMra1412877, indexed in Pubmed: 26422725.

7. Holliday MA, Ray PE, Friedman AL. Fluid therapy for children: facts, fashions and questions. Arch Dis Child. 2007; 92(6): 546-550, doi: 10.1136/adc.2006.106377, indexed in Pubmed: 17175577.

8. Ray PE. Neurological complications from dysnatremias in children: a different point of view. Pediatr Nephrol. 2006; 21(7): 1048-9; author reply 1050, doi: 10.1007/s00467-006-0111-9, indexed in Pubmed: 16773423.
9. Van Regenmortel N, DeWeerdt T, Van Craenenbroeck AH, et al. Effect of isotonic versus hypotonic maintenance fluid therapy on urine output, fluid balance, and electrolyte homeostasis: a crossover study in fasting adult volunteers. Br J Anaesth. 2017; 118(6): 892-900, doi: 10.1093/bja/ aex118, indexed in Pubmed: 28520883.

10. Lobo DN, Bostock KA, Neal KR, et al. Effect of salt and water balance on recovery of gastrointestinal function after elective colonic resection: a randomised controlled trial. Lancet. 2002; 359(9320): 1812-1818, doi: 10.1016/S0140-6736(02)08711-1, indexed in Pubmed: 12044376.

11. Levey AS, Stevens LA, Schmid CH, et al. CKD-EPI (Chronic Kidney Disease Epidemiology Collaboration). A new equation to estimate glomerular filtration rate. Ann Intern Med. 2009; 150(9): 604-612, indexed in Pubmed: 19414839.

12. National Institute for Health and Care Excellence Guideline for Intravenous fluid therapy in adults in hospital (CG174). In: National Institute for Health and Care Excellence Guideline for Intravenous fluid therapy in adults in hospital (CG174). : National Institute for Health and Care Excellence Guideline for Intravenous fluid therapy in adults in hospital . 2013.

13. Kellum J, Elbers PW. Stewart's Textbook of Acid-Base: Acidbase.org; 2009.

\section{Corresponding author:}

Niels Van Regenmortel

Department of Intensive Care Medicine

Antwerp University Hospital

Wilrijkstraat 10, B2640, Edegem, Belgium

e-mail:niels.vanregenmortel@uza.be

Received: 17.10.2017

Accepted: 23.11.2017 